\title{
Hemicraniotomy in Massive Hemispheric Stroke: A Stark Perspective on a Radical Procedure
}

\author{
Eelco F. M. Wijdicks
}

Can. J. Neurol. Sci. 2000; 27: 271-273

Medical management of progressive brain swelling in patients after a massive hemispheric stroke has been discouraging. Traditional medical interventions that reduce intracranial pressure (ICP) at the time of profound swelling, such as hyperventilation, mannitol, and barbiturates, have remained unsuccessful. ${ }^{1,2}$ The mechanism of clinical deterioration is more readily explained by a gradual growing ischemic swollen mass compressing structures and brain stem and less likely through a mechanism of globally increased ICP and decreased cerebral perfusion pressure. Decompressive craniectomy has been revived as a surgical treatment of massive hemispheric stroke associated with brain swelling. The surgical procedure involves removal of the skull, durotomy and insertion of a cadaveric dura to facilitate further stretch from the abutting swollen hemisphere. The swollen brain remains untouched and removal of necrotic tissue or limited lobectomy is deferred. The temporary removal of a large part of the skull on one side, leaving frontal and occipital portions in place is, as expected, an intimidating proposal to the patient's spouse or kin. The modified procedure has been spearheaded by Hacke ${ }^{3}$ and associates in Germany and has found followers in the United States but is not routinely performed in many European countries, United Kingdom, Canada and Australia. I continue to find it difficult to obtain consent for the procedure, particularly in elderly patients who have already cemented their wishes in a living will, and I am very concerned that a future randomized trial may not provide us with a desirable result. From the beginning, these unfortunate patients have a very bad stroke, only a portion will be selected for the procedure and a major handicap in survivors may be inevitable.

\section{MIDDLE CEREBRAL ARTERY TERRITORY INFARCTION AND ITS TIME COURSE}

An occlusion of the stem of the middle cerebral artery causes a devastating ischemic insult to one hemisphere. It leads to hemianopsia, flaccid hemiplegia of the arm with some movement of the leg, gaze deviation to the affected hemisphere, and global aphasia in the left middle cerebral territory and neglect in the left body parts if the right side is affected. A cardioembolic source is often implicated but, in younger individuals carotid artery dissection is more prevalent. ${ }^{4} \mathrm{CT}$ scanning is able to document the evolution of infarction fairly early after presentation, although major discrepancies between the initial CT scan and clinical findings are common.
Approximately half of the patients who present with a large hemispheric infarct will deteriorate clinically. Evolution and further maturation of the infarct may cause deterioration. In others, a decrease in their level of consciousness will be noted, and when it emerges it is associated with the development of swelling in the affected hemisphere. Occasionally reperfusion hemorrhage will cause a large intracranial hematoma causing signs of brain herniation. The clinical progression of these infarcts may take many forms and includes rapidly evolving uncal herniation, gradual unrelenting deterioration with signs of diencephalic herniation, but also drowsiness for five to seven days without need for airway protection through intubation and even improvement from the ictus. The chance of brain edema developing is clearly related to the size of infarction, particularly with more than 50 percent area involvement on CT (either hypodensity or sulci effacement) within six hours of presentation and a minimum baseline of 15 on the National Institute of Health Stroke Score. ${ }^{5}$

An influential study by Von Kummer ${ }^{6}$ and associates in an ICU population found that the emergence of an early hypodensity involving more than 50 percent of the distribution of the middle cerebral artery or local brain swelling may lead to an 80 percent mortality. This study could have been influenced by referral bias involving the most severely affected patients in the intensive care unit. ${ }^{6}$ In our hospital-based series of patients, including patients initially admitted to the ward, mortality was 70 percent in large hemispheric stroke only if deterioration occurred beyond drowsiness and associated with developing signs of early uncal or diencephalic herniation. ${ }^{7}$ We have not only been struck by the fact that several patients with massive swelling and shift on CT scan do not progress further clinically, but also are younger. ${ }^{7}$ This significant decrease in mortality in younger patients has its explanation in a less severe brain swelling. Measuring CT parameters for shift and brain stem displacement, brain swelling was much less severe in younger patients, although they do have less space to accommodate for a swollen ischemic mass. ${ }^{7}$ Why young individuals (in our study defined as less than 45 years old) develop less severe edema has

From the Department of Neurology, Neurological-Neurosurgical Intensive Care Unit, St. Marys Hospital, Mayo Clinic and Foundation, Rochester, MN

ReCEIVED JANuARy 24, 2000. ACCEPTEdin FinAlform August 22, 2000 Reprint requests to: E.F.M. Wijdicks, Mayo Clinic, W8B, Department of Neurology, 200 First Street, SW, Rochester, MN 55905 


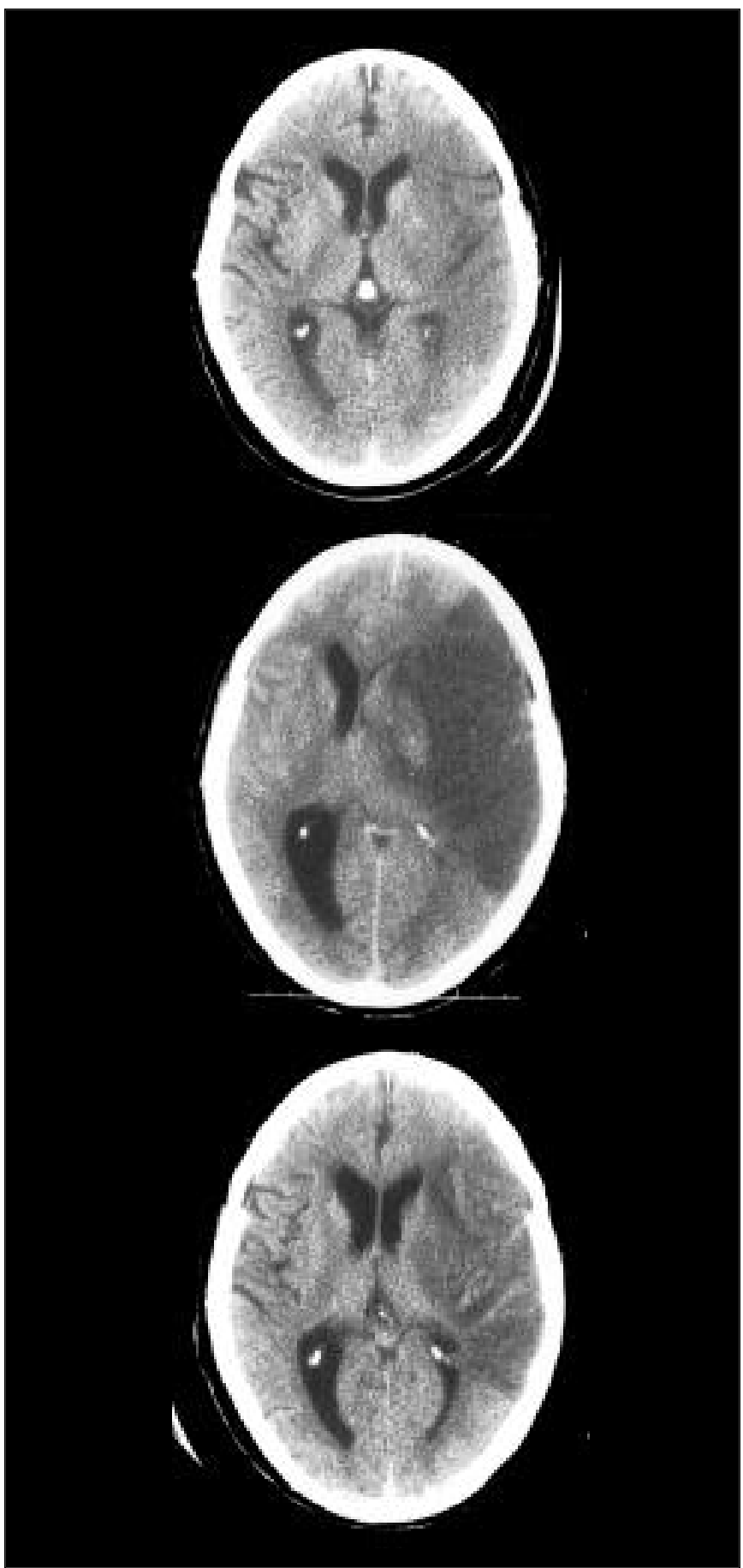

Figure: Serial CT scan in 62-year-old male showing the evolution of a middle cerebral artery infarct with brain swelling.

TOP ROW: Admission CTscan showing effacement of sulci in the entire MCA territory of the dominant hemisphere. The outer rim of the lentiform nucleus is also effaced. MIDDLE ROW: Two days later, development of a large swollen ischemic mass with compression of the frontal horn and shift of the septum pellucidum. LOWER ROW: CTscan five days from the ictus without any medical or surgical intervention (family declined craniotomy) showing marked improvement of the swelling. This patient while having a devastating global aphasia and left hemiparesis only had brief periods of decreased level of consciousness but continued to localize to pain and occasionally followed commands and was able to protect his airway. At his last CT scan he was alert. not yet been elucidated, although one study suggests a better reactivity to vasodilator stimuli or relative deficiency of collateral circulation with aging. ${ }^{8}$

It is thus troubling that the published surgical cohorts that have gathered up to hundreds of patients are skewed towards younger patients and some series have involved younger patients four times more frequently. ${ }^{9}$ As alluded to earlier, I assume the rationale to perform the procedure in younger patients has been based on the assumption that younger patients are less able to tolerate brain edema. The natural history can be favorable despite a series of CT scan portending unrelentless swelling (Figure). Hacke et $\mathrm{al}^{3}$ correctly pointed out the presence of a group of patients with rapidly developing edema leading to endstage herniation within days of onset, sometimes within 36 hours. This so-called "malignant middle cerebral artery infarct" may be much less frequent than previously claimed and may be difficult to identify early. Results from cohorts representing the most severe cases should not be extrapolated to the entire population which may have a much more favorable natural history. Population studies are not available and I suspect may demonstrate a less gloomier prognosis than 80 percent mortality.

\section{Is A TriaL JUSTIFIED?}

When we, at the risk of presumption, extrapolate the available published data to the entire population of patients, we may expect that approximately one in two patients with a large middle cerebral infarct and early hypodensity on CT scan (an arbitrary selection) will deteriorate. If decompressive craniotomy is done based on early clinical findings and early CTscan abnormalities, one would expect that decompressive craniectomy will only be successful in the category of patients who deteriorate beyond drowsiness. Thus, if a trial would be devised purely based on Glasgow coma score and CT scan findings and not on definitive clinical signs of herniation, the reduction in mortality may be much less than expected. Another major concern, inherent to surgical trials, is whether postoperative care is similar to that in patients left untreated. The mind set and core skills of neurocritical care of treating physicians could be different in untreated patients resulting, for example, in less aggressive postoperative surveillance leading to increased mortality in nonsurgical patients. Withdrawal of support may be an early consideration in untreated patients and family enthusiasm for maximal support may change overnight. Whether neurologic morbidity is decreased by early decompressive hemicraniectomy is uncertain, and we suspect that many patients will remain in a helpless crippled state. In addition, without blinding of neurologists who have to grade handicap, accuracy of data collection may be seriously impacted. Therefore, the results of such a trial may be fractured with some reduction in mortality but major disability in survivors, leaving its synthesized value for daily practice almost nil. Very early hemicraniotomy based on CT scan only, may result in no difference in outcome or possibly a minor shift in disability due to less damaging effect of swelling. Late surgery can only lead to reduction in mortality but possibly leaving a survivor with only a shred of cognitive function. My argument is that carefully planned natural history studies documenting the different types of deterioration using serial CT scans are needed. In addition, MR may become useful in predicting and selecting patients for surgery. Despite failure to 
influence outcome with medical measures at the height of brain swelling, some untried possibilities remain, for example, use of a preemptive strike with osmotic diuretic agents ${ }^{10}$ or the use of invasive ultrafiltration catheters to remove brain edema osmotically.

Nevertheless, cynicism should not take over idealism. Hemicraniotomy with duraplasty should be given a chance and anticipate these pitfalls resulting in careful matching of age and postoperative care. A trial (HEADSTART), is currently underway.

\section{REFERENCES}

1. Wijdicks EFM. Management of massive hemispheric cerebral infarct: is there a ray of hope? Mayo Clin Proc 2000; 75:945-952.

2. Manno EM, Adams RE, Deyn CP, et al. The effects of mannitol in cerebral edema after large hemispheric cerebral infarct. Neurology 1999;52:583-587.

3. Hacke W, Schwab S, Horn M, et al. Malignant middle cerebral territory infarction: clinical course and prognostic signs. Arch Neurol 1996;53:309-315.
4. Heinsius T, Bogousslavsky J, Van Melle G. Large infarcts in the middle cerebral artery territory. Etiology and outcome patterns. Neurology 1998;50:341-350.

5. Krieger DW, Demchule AM, Kasner SE. Early clinical and radiological predictors of fatal brain swelling in ischemic stroke. Stroke 1999;30:287-292.

6. Von Kummer R, Meyding-Lamade U, Forsting M, et al. Sensitivity and prognostic value of early compiled tomography in occlusion of the middle cerebral artery trunk. AJNR Am J Neuroradiol 1994;15:9-15.

7. Wijdicks EFM, Diringer MN. Middle cerebral artery territory infarction in early brain swelling: progression and effective age on outcome. Mayo Clin Proc 1998;73:829-836.

8. Davis M, Mendelow AD, Perry RH, Chambers IK, James OF. The effect of age on cerebral edema, cerebral infarction and neuroprotective potential in experimental occlusive stroke. Acta Neurochir Suppl. (Wien) 1999:60:282-284.

9. Schwab S, Steiner T, Ashoff A, et al. Early hemicraniotomy in patients with complete middle cerebral artery infarction. Stroke 1998;29:1888-1893.

10. Wijdicks EFM, Schievink WI, McGough PF. Dramatic reversal of uncal syndrome in brain edema from infarction in the middle cerebral artery territory. Cerebrovasc Dis 1997;7:349-352. 